\title{
CAPACITY REGION OF THE MULTIANTENNA GAUSSIAN BROADCAST CHANNEL WITH ANALOG TX-RX BEAMFORMING
}

\author{
Ignacio Santamaría, Javier Vía, Alfredo Nazábal, Christian Lameiro \\ Communications Engineering Dept., University of Cantabria, Santander, Spain. \\ e-mail: \{nacho,jvia,alfredo\}@gtas.dicom.unican.es
}

\begin{abstract}
In this paper we characterize the capacity boundary of the two-user multiple-input multiple-output (MIMO) broadcast channel (BC) when the multiantenna terminals use analog beamforming at both sides of the link. Basically, the problem reduces to finding the optimal transmit direction and the optimal power allocation between users to operate at any point on the boundary. We show that both optimization problems can be solved in closed form. First, exploiting the fact that any Pareto optimal pair of rates must also be Pareto optimal of a convex region defined by the channel energies, the optimal transmit direction is shown to be the principal eigenvector of a matrix. Second, the optimal power allocation is obtained by exploiting again the Pareto optimality of the sought pair of rates. Although this paper focuses on the $\mathrm{BC}$, the obtained results also serve to characterize the dual multiple-access channel (MAC). Moreover, this explicit characterization of the boundary can be extended to a $\mathrm{K}$-user system for $\mathrm{K}>2$. Numerical examples are provided to illustrate the results of the paper, and to show the enlargement of the capacity region achieved by analog beamforming in comparison to a single-input single-output (SISO) BC.
\end{abstract}

Index Terms - Broadcast channel, analog beamforming, Pareto boundary.

\section{INTRODUCTION}

Multiple-input multiple-output (MIMO) wireless radios that apply analog beamforming techniques already in the radio frequency domain (denoted in this paper as RF-MIMO) have recently received renewed interest due to their reduced system size, cost and power consumption in comparison to conventional MIMO systems [1], [2].

An RF-MIMO receiver (the transmitter operates analogously) applies a set of complex weights $\mathbf{w}_{R}=\left(w_{1}, \ldots, w_{n_{R}}\right)$, each weight representing a gain factor and phase shift, to the received RF signals as shown in Fig. 1. In this way, after combining the weighted RF signals, a single stream of data must be acquired and processed and thus the hardware cost and the power consumption are significantly reduced. Although the multiplexing gain of an RFMIMO transceiver is limited to one (since we transmit/receive a single data stream), in [2] we have shown that other benefits of the MIMO channel such as full spatial diversity or full array gain can be retained by the proposed architecture if proper processing is carried out.

For point-to-point links, the design of the optimal Tx-Rx analog beamformers for multicarrier transmissions has been thoroughly considered in [3]-[5]. In this paper, we extend the study to the downlink or broadcast channel (BC) where one multiantenna transmitter that applies analog beamforming wishes to send different

The research leading to these results has received funding from the European Community's Seventh Framework Programme (FP7/2007-2013) under grant agreement n 213952, MIMAX and by the Spanish Government (MICINN) under projects TEC2007-68020-C04-02/TCM (MULTIMIMO) and CONSOLIDER-INGENIO 2010 CSD2008-00010 (COMONSENS).

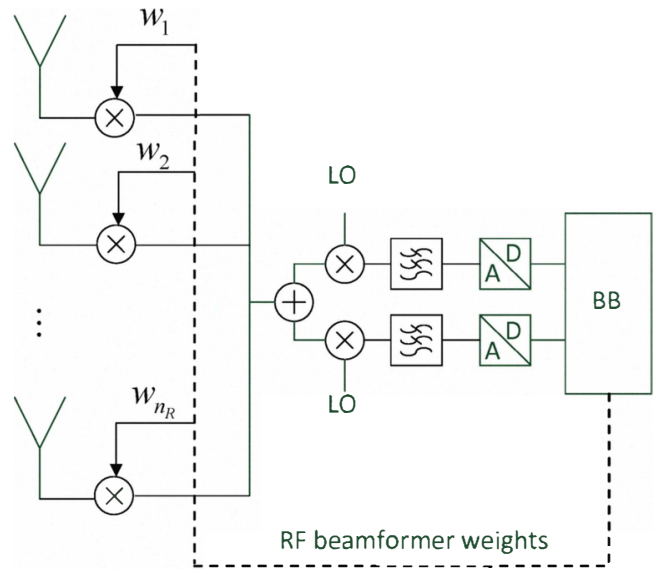

Fig. 1. Analog antenna combining in the RF path for MIMO communications systems (RF-MIMO).

data streams to different receivers also equipped with analog beamformers as shown in Fig. 2.

The BC with single antenna terminals is well-known in the information theory literature [6], [7], and its capacity region boundary is achieved by superposition coding at the transmitter with successive interference cancellation at the receivers. In the MIMO case with conventional receivers (i.e., as many downconversion/upconversion chains as signal paths), the BC capacity region was shown to be achievable using dirty paper coding [8], [9]. Nevertheless, the analog beamforming architecture introduces some restrictions which make the problem new and worth of study. For instance, the RF circuitry used for analog combining, which typically consists of a vector modulator [10], is not able to change the analog beamformer weights at the symbol rate. From a practical point of view this means that the beamformers must be kept fixed during the transmission of a whole data frame. As a consequence of this hardware limitation, time-sharing arguments cannot be used to characterize the rate region of this particular broadcast channel.

In the paper we show that the Pareto optimal points of the rate region can be achieved by transmitting with superposition coding through a given direction. At the receiver side the users apply maximum ratio beamforming and successive cancellation. Most interestingly, for any operating point on the boundary the optimal transmit direction and the power distribution between users can be obtained in closed form. In this way we are able to provide an explicit characterization of the rate region boundary. Although in this paper we mainly focus on the two-user BC, the derived results also serve to characterize the multiple-access channel (MAC), since a duality relationship also holds for this channel model. Moreover, the proposed procedure can be easily extended to the K-user case. 


\section{PROBLEM FORMULATION}

Let us consider for simplicity the two-user BC depicted in Fig. 2. It consists of a base station with $n_{T}$ antennas transmitting a single data stream $s[n]$ that encodes the information intended for both users (e.g., by superposition coding) through the analog beamformer $\mathbf{w}_{T} \in \mathbb{C}^{n_{T} \times 1}$. The total power at the base station is normalized to $1\left(E\left[|s[n]|^{2}\right]=1\right.$ and $\left.\left\|\mathbf{w}_{T}\right\|^{2}=1\right)$. The transmitted signal at time instant $n$ is then given by

$$
\mathbf{x}=\mathbf{w}_{T} s[n] .
$$

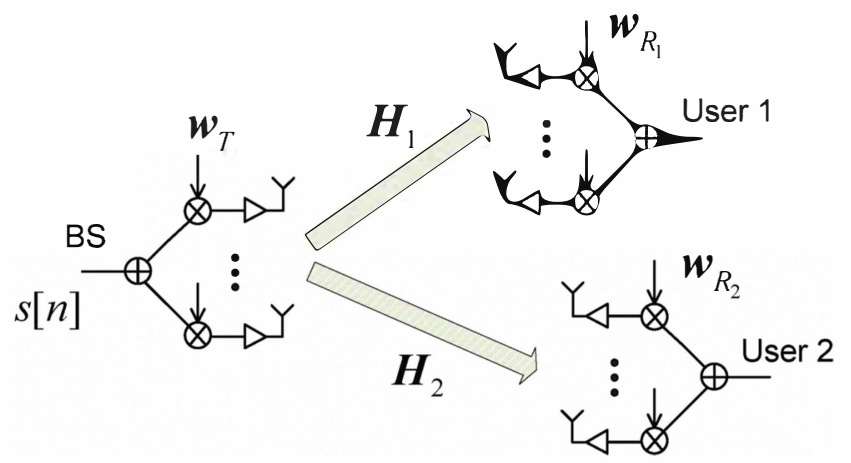

Fig. 2. Two-user multiantenna broadcast channel with analog beamforming.

On the receiver side both users have $n_{R}$ antennas ${ }^{1}$ and also perform analog combining with beamformers $\mathbf{w}_{R_{1}} \in \mathbb{C}^{n_{R} \times 1}$ and $\mathbf{w}_{R_{2}} \in \mathbb{C}^{n_{R} \times 1}$. Therefore, the signal received by the $i$-th user is given by

$$
\mathbf{y}_{i}=\mathbf{w}_{R_{i}}^{H} \mathbf{H}_{i} \mathbf{w}_{T} s[n]+\mathbf{w}_{R_{i}}^{H} \mathbf{r}_{i}, \quad i=1,2 ;
$$

where the $n_{R} \times n_{T}$ MIMO channels, $\mathbf{H}_{1}$ and $\mathbf{H}_{2}$, are assumed to be flat-fading (i.e., frequency non-selective), and $\mathbf{r}_{i}$ is the additive noise vector whose components are i.i.d. zero-mean circularly complex Gaussian random variables with variance $\sigma^{2}$. Finally, both channels are assumed to be perfectly known at the transmitter.

The analog beamforming architecture introduces some restrictions that make the problem of characterizing its rate region worth of study. To the best of our knowledge, this problem has not been considered in the information theory literature. Specifically, one of the distinguishing features of the analog beamforming topology is that, due to the limitations of the associated RF circuitry, the complex weights in the RF path change at a much slower rate than the symbol rate. In practice, this means that the $\mathrm{Tx}^{-}$ $\mathrm{Rx}$ beamdirections must be kept fixed during the transmission of a whole data frame. This limitation has several interesting implications. First, time-sharing arguments, which are typically invoked to solve many rate region problems, cannot be used here. Second, conventional MIMO beamforming approaches that transmit different streams intended for different users through different directions (e.g., $\mathbf{x}=\mathbf{w}_{T_{1}} s_{1}[n]+\mathbf{w}_{T_{2}} s_{2}[n]$ ) cannot be applied either. Consequently, the many algorithms proposed to jointly find optimal beamformers and power control in downlink MIMO channels [11], [12] are of no application to our problem, since they would require to change the analog beamformer weights in a symbol-by-symbols basis.

With this setting, the most interesting question from a theoretical point of view aims at characterizing the capacity region and,

\footnotetext{
${ }^{1}$ The generalization to users with different number of receive antennas is straightforward.
}

specially, its boundary for this particular broadcast channel. This question will be answered in the next sections.

\section{CAPACITY REGION}

For a fixed transmit beamformer, the system reduces to a downlink channel with a single transmit antenna and multiple receive antennas at each user. This channel is degraded [6] and the optimal strategy for both users is matched filtering with respect to their channels [13]. Therefore, the unit-norm optimal analog receive beamformers are

$$
\mathbf{w}_{R_{i}}=\mathbf{H}_{i} \mathbf{w}_{T} /\left\|\mathbf{H}_{i} \mathbf{w}_{T}\right\|, \quad i=1,2 .
$$

After matched filtering, the signal received by each user is

$$
\mathbf{y}_{i}=h_{i} s[n]+r_{i}, \quad i=1,2 ;
$$

where we have introduced the effective (real) SISO channels $h_{i}=\sqrt{\mathbf{w}_{T}^{H} \mathbf{H}_{i}^{H} \mathbf{H}_{i} \mathbf{w}_{T}}$, which only depend on the Tx beamformer, and $r_{i}$ is a zero-mean Gaussian random variable with variance $\sigma^{2}$. Therefore, for any fixed transmit direction $\mathbf{w}_{T}$, the MIMO BC with analog beamforming reduces to a SISO BC for which the capacity achieving scheme is superposition coding [13], [14]. In particular, assuming that $h_{1}>h_{2}$, the two-user capacity region of the SISO BC using superposition coding and successive interference cancellation is the pair of rates [7]

$$
\begin{array}{r}
C_{B C}^{S I S O}\left(\mathbf{w}_{T}\right)=\bigcup_{0 \leq P \leq 1}\left(R_{1}=\log _{2}\left(1+\frac{P E_{1}}{\sigma^{2}}\right),\right. \\
\left.R_{2}=\log _{2}\left(1+\frac{(1-P) E_{2}}{P E_{2}+\sigma^{2}}\right)\right),
\end{array}
$$

where $E_{i}=h_{i}^{2}=\mathbf{w}_{T}^{H} \mathbf{H}_{i}^{H} \mathbf{H}_{i} \mathbf{w}_{T}$ is the channel energy of the effective SISO channel for the $i$-th user, and the rates are in units of bits/s/Hz. In (2) we have made explicit the dependence of the rate region with the transmit beamforming.

The explanation of (2) is as follows. The transmitted signal $s[n]$ is constructed as a linear superposition of the signals for both users with a total unit-power constraint. Since $h_{1}>h_{2}$, user 1 can decode any data transmitted to user 2 ; therefore user 1 decodes and cancels user 2's message and then decodes its own message. On the other hand, user 2 has a worse SISO channel and treats the signal of user 1 as noise. This scheme gives the rates $R_{1}$ and $R_{2}$ in the boundary of (2) without time sharing.

It is then clear that the achievable rate region for the multiantenna broadcast channel with analog Tx-Rx beamforming is given by the union of the SISO BC rate regions obtained by changing the unitnorm transmit beamformer: $C_{B C}^{S I S O}\left(\mathbf{w}_{T}\right)$. More formally,

$$
C_{B C}^{R F-M I M O}=\bigcup_{\left\|\mathbf{w}_{T}\right\|=1} C_{B C}^{S I S O}\left(\mathbf{w}_{T}\right)
$$

Although the individual SISO BC regions are convex, notice that the union in (3) is not necessarily convex. In fact, it can be concave for low signal-to-noise ratios $(S N R)^{2}$. This is a remarkable difference with other theoretical results related to BC channels. Obviously, (3) could be convexified if time-sharing is allowed, but as we have previously remarked the RF-MIMO architecture prevents any form of time-sharing.

Some points of the boundary can be easily obtained; for instance, to operate at the maximum sum-rate point we have to transmit all the available power to the best user along its strongest eigenmode. That is, assuming that $h_{1}>h_{2}, \mathbf{w}_{T}$ must be the eigenvector corresponding to the largest eigenvalue of $\mathbf{H}_{1}^{H} \mathbf{H}_{1}$ : this scheme is well-known in the MIMO literature and it is typically referred to as dominant eigenmode transmission or DET [15].

${ }^{2}$ Depending on the $S N R$ and the MIMO channels, the region (3) is either convex or concave. 
Fig. 3 exemplarily depicts the capacity curves $C_{B C}^{S I S O}\left(\mathbf{w}_{T}\right)$ in (2) for several randomly generated transmit beamformers for a twouser RF-MIMO broadcast channel in which all terminals have 4 antennas. The signal-to-noise ratio $(S N R)$ for users 1 and 2 is $S N R_{1}=10 \mathrm{~dB}$ and $S N R_{2}=0 \mathrm{~dB}$, respectively. We have also included in solid line the DET curves for both users. The outer boundary of all these curves is the Pareto boundary, which gives us the set of rate points at which it is impossible to increase any of the rates without decreasing the other. Any point of the Pareto boundary is characterized by a pair $^{3}\left(\mathbf{w}_{T}^{*}, P^{*}\right)$ whose elements are the optimal transmit beamformer and the power assigned to user 1 . As we will show in the next section, these Pareto optimal points defining the boundary can be obtained in closed form.

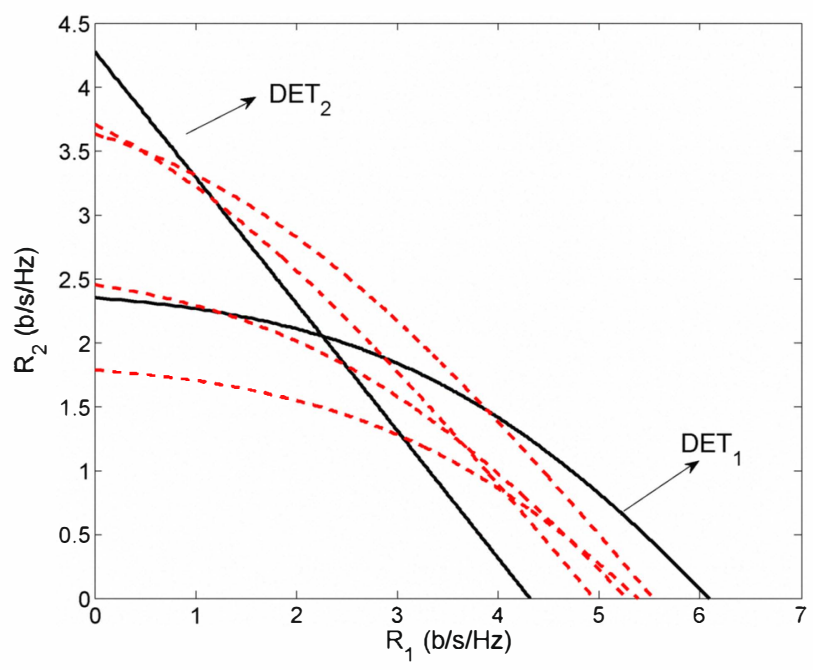

Fig. 3. Capacity regions for the two-user RF-MIMO broadcast channel with different transmit beamformers $\left(S N R_{1}=10 \mathrm{~dB}\right.$ and $S N R_{2}=0 \mathrm{~dB}$ ). The black solid lines depict the rate curves corresponding to dominant eigenmode transmission (DET) for users 1 and 2. The rest of curves have been obtained with Tx beamformers randomly generated.

\section{CHARACTERIZATION OF THE CAPACITY BOUNDARY}

\section{IV-A. Optimal transmit beamforming}

The following theorem proves that the optimal transmit beamformers (i.e., those achieving rate pairs on the boundary of the capacity region) can be obtained in closed form by maximizing a weighted sum of the equivalent SISO channel energies.

Theorem 1: The points on the Pareto boundary of the rate region, i.e., $\left(R_{1}^{*}, R_{2}^{*}\right)$, are achievable by transmit beamformers that are solution of the following maximization problem

$$
\max _{\mathbf{w}_{T}} \alpha E_{1}+(1-\alpha) E_{2},
$$

for some $0 \leq \alpha \leq 1$.

Proof: Let us first define the energy region $\left\{\left(E_{1}, E_{2}\right) \mid E_{1}=\right.$ $\left.\mathbf{w}_{T}^{H} \boldsymbol{\Sigma}_{1} \mathbf{w}_{T}, E_{2}=\mathbf{w}_{T}^{H} \boldsymbol{\Sigma}_{2} \mathbf{w}_{T}\right\}$, where we have defined $\boldsymbol{\Sigma}_{i}=$ $\mathbf{H}_{i}^{H} \mathbf{H}_{i}$. This region is convex and therefore the Pareto optimal

\footnotetext{
${ }^{3}$ Throughout this paper and with some abuse of notation, the superscript * will be used to denote both the Pareto optimal points or the parameters (i.e., beamformer and power) used to achieve it.
}

points on its boundary can be obtained by solving, for $0 \leq \alpha \leq 1$, the weighted sum-energy maximization problem in (4) [16].

The proof now goes by contradiction. Assume that $E_{1}=\left(\mathbf{w}_{T}^{*}\right)^{H} \boldsymbol{\Sigma}_{1} \mathbf{w}_{T}^{*}$ and $E_{2}=\left(\mathbf{w}_{T}^{*}\right)^{H} \boldsymbol{\Sigma}_{2} \mathbf{w}_{T}^{*}$ are not Pareto optimal of the energy region. Therefore, there must exist a different transmit beamformer $\mathbf{w}_{T}^{\prime}$ that, for instance, improves $E_{1}$ without lowering $E_{2}$. Since $R_{1}$ and $R_{2}$ are monotonic increasing functions of $E_{1}$ and $E_{2}$, respectively, this necessarily means that $R_{1}^{\prime}>R_{1}^{*}$ while $R_{2}^{\prime}=R_{2}^{*}$ and consequently $\left(R_{1}^{*}, R_{2}^{*}\right)$ cannot be Pareto optimal of the rate region, which concludes the proof.

Remark 1: Theorem 1 only proves that all beamformers that are Pareto optimal in rates are also optimal in terms of channel energies. However, the implication on the other direction is not always true. That is, all beamformers that are Pareto optimal in terms of energies are not necessarily optimal in terms of rate pairs. This remark will be clarified later with some examples.

Remark 2: The usefulness of Theorem 1 is that the optimal transmit beamformers can be obtained in closed form as the main eigenvector of the $n_{T} \times n_{T}$ positive semidefinite matrix $\boldsymbol{\Sigma}_{\alpha}=\alpha \boldsymbol{\Sigma}_{1}+(1-\alpha) \boldsymbol{\Sigma}_{2}$ when $\alpha$ varies from 0 to 1 . To illustrate this idea, we have simulated a two-user RF-MIMO BC with 4 antennas at both sides of the link. The channel of user 1 is $20 \mathrm{~dB}$ better than that of user 2. For this scenario, we have obtained the optimal transmit beamformer, $\mathbf{w}_{T}^{*}$, that maximizes the weighted energy cost function (4) with $\alpha=0.01$. The capacity region of the resulting SISO BC is shown in Fig. 4 with red dotted line. Additionally, the boundary of $C_{B C}^{R F-M P M O}$ is depicted in black solid line. We observe that there is an optimal power, $P^{*}$, for which the SISO boundary is tangent to the RF-MIMO boundary, that is, $\left(\mathbf{w}_{T}^{*}, P^{*}\right)$ is Pareto optimal. To complete the characterization of the boundary of $C_{B C}^{R F-M I M O}$, we have to devise a method to find the optimal power allocation, $P^{*}$, that yields the tangency point in Fig. 4. As we will show in the next subsection, this also can be obtained in closed form.

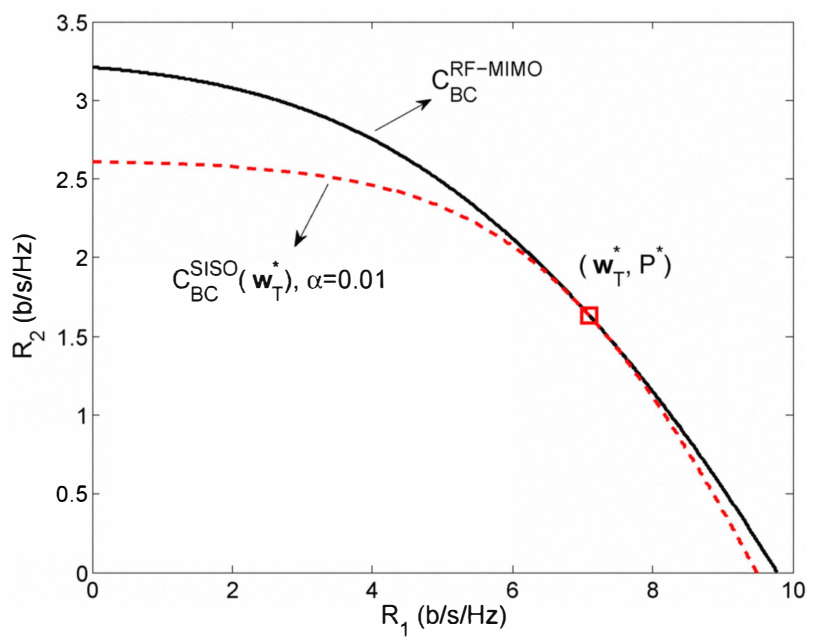

Fig. 4. The red dotted line depicts the capacity region of the SISO BC, $\left(C_{B C}^{S I S O}\right)$, corresponding to an optimal transmit beamformer obtained by solving (4) with $\alpha=0.01\left(S N R_{1}=20 \mathrm{~dB}\right.$ and $S N R_{2}=0 \mathrm{~dB}$ ). The black solid line shows $C_{B C}^{R F-M I M O}$. The square marker indicates the point of tangency. 


\section{IV-B. Optimal power allocation}

The key idea to obtain $P^{*}$ consists in exploiting the Pareto optimality of the sought point $\left(\mathbf{w}_{T}^{*}, P^{*}\right)$, where $\mathbf{w}_{T}^{*}$ is a maximizer of (4) for a given $\alpha$. To develop this idea let us consider separately the $\operatorname{cases}^{4} h_{1}^{*}>h_{2}^{*}$ and $h_{2}^{*}>h_{1}^{*}$.

Case $h_{1}^{*}>h_{2}^{*}$ : In this case the SISO BC boundary is given by the following pair of rates

$$
\begin{aligned}
& R_{1}=\log _{2}\left(1+\frac{P E_{1}^{*}}{\sigma^{2}}\right) \\
& R_{2}=\log _{2}\left(1+\frac{(1-P) E_{2}^{*}}{P E_{2}^{*}+\sigma^{2}}\right),
\end{aligned}
$$

for $0 \leq P \leq 1$.

The power assigned to user 1 can be written in terms of $R_{1}$ as

$$
P=\gamma_{1} \sigma^{2} / E_{1}^{*},
$$

where $\gamma_{1}=\left(2^{R_{1}}-1\right)$. Substituting (7) into (6), the rate achieved by user 2 can be expressed as

$$
R_{2}=\log _{2}\left(1+\frac{\left(E_{1}^{*}-\gamma_{1} \sigma^{2}\right) E_{2}^{*}}{\gamma_{1} \sigma^{2} E_{2}^{*}+\sigma^{2} E_{1}^{*}}\right) .
$$

The Pareto optimality of $P^{*}$ means that, for $R_{1}$ fixed, there does not exist any other unit-norm transmit beamforming $\mathbf{w}_{T}^{\prime} \neq \mathbf{w}_{T}^{*}$ such that $R_{2}^{\prime}>R_{2}^{*}$. Therefore, $P^{*}$ can be obtained by equating to zero the gradient of $R_{2}$ with respect to $\mathbf{w}_{T}^{*}$ and subject to $\left\|\mathbf{w}_{T}^{*}\right\|^{2}=1$. The unconstrained gradient of (8) with respect to $\mathbf{w}_{T}^{*}$ is given by

$$
\begin{aligned}
\nabla_{\mathbf{w}_{T}^{*}} R_{2} & =\frac{1}{K}\left(\gamma_{1}\left(\left(E_{2}^{*}\right)^{2} \boldsymbol{\Sigma}_{1}-\sigma^{2} E_{1}^{*} \boldsymbol{\Sigma}_{2}+\sigma^{2} E_{2}^{*} \boldsymbol{\Sigma}_{1}\right) \mathbf{w}_{T}^{*}\right. \\
& \left.+\left(E_{1}^{*}\right)^{2} \boldsymbol{\Sigma}_{2} \mathbf{w}_{T}^{*}\right) .
\end{aligned}
$$

where $K=\left(\gamma_{1}+1\right) \sigma^{2}\left(\gamma_{1} E_{2}^{*}+E_{1}^{*}\right)^{2} / 2$ is a positive number.

The unconstrained gradient, however, cannot be directly equated to zero in order to get $\gamma_{1}$ since it depends on the norm of the transmit beamformer. To find the optimal $\gamma_{1}$ and therefore the optimal $P$ through (7), we have to compute the gradient of (8) on the Stiefel manifold consisting of all unit-norm complex $n_{T} \times 1$ beamformers. According to [17], the gradient on the Stiefel manifold is the vector on the tangent space, $\Delta R_{2}$, given by

$$
\Delta R_{2}=\left(\mathbf{I}-\mathbf{w}_{T}^{*}\left(\mathbf{w}_{T}^{*}\right)^{H}\right) \nabla_{\mathbf{w}_{T}^{*}} R_{2} .
$$

Equating (10) to zero and taking (7) into account we finally find that the optimal $P^{*}$ can be expressed in closed form as ${ }^{5}$

$$
P^{*}=\frac{\sigma^{2}\left\|\mathbf{a}_{1}\right\|}{E_{1}^{*}\left\|\mathbf{b}_{1}\right\|},
$$

where $\mathbf{a}_{1}$ and $\mathbf{b}_{1}$ are $n_{T} \times 1$ vectors given by

$$
\mathbf{a}_{1}=\left(\mathbf{I}-\mathbf{w}_{T}^{*}\left(\mathbf{w}_{T}^{*}\right)^{H}\right)\left(\left(E_{2}^{*}\right)^{2} \boldsymbol{\Sigma}_{1}-\sigma^{2} E_{1}^{*} \boldsymbol{\Sigma}_{2}+\sigma^{2} E_{2}^{*} \boldsymbol{\Sigma}_{1}\right) \mathbf{w}_{T}^{*}
$$

and

$$
\mathbf{b}_{1}=\left(\mathbf{I}-\mathbf{w}_{T}^{*}\left(\mathbf{w}_{T}^{*}\right)^{H}\right)\left(E_{1}^{*}\right)^{2} \boldsymbol{\Sigma}_{2} \mathbf{w}_{T}^{*}
$$

respectively.

\footnotetext{
${ }^{4}$ When $h_{1}^{*}=h_{2}^{*}$, both cases give identical results.

${ }^{5}$ The same result could have been obtained by introducing the unit-norm constraint by means of a Lagrange multiplier and analyzing the KarushKuhn-Tucker (KKT) conditions [16].
}

Case $h_{2}^{*}>h_{1}^{*}$ : When the user 2 has a better channel than the user 1 , the achievable rates are the following

$$
\begin{aligned}
& R_{2}=\log _{2}\left(1+\frac{(1-P) E_{2}^{*}}{\sigma^{2}}\right), \\
& R_{1}=\log _{2}\left(1+\frac{P E_{1}^{*}}{(1-P) E_{1}^{*}+\sigma^{2}}\right),
\end{aligned}
$$

for $0 \leq P \leq 1$. Fixing the rate of user 2 , the relationship between $P$ and $\gamma_{2}=\left(2^{R_{2}}-1\right)$ is now given by

$$
P=1-\frac{\gamma_{2} \sigma^{2}}{E_{2}^{*}} .
$$

Taking the gradient of $R_{1}$ on the Stiefel manifold and equating it to zero, we obtain the optimal power for user 1 as

$$
P=1-\frac{\sigma^{2}\left\|\mathbf{a}_{2}\right\|}{E_{2}^{*}\left\|\mathbf{b}_{2}\right\|},
$$

where

$$
\mathbf{a}_{2}=\left(\mathbf{I}-\mathbf{w}_{T}^{*}\left(\mathbf{w}_{T}^{*}\right)^{H}\right)\left(\left(E_{1}^{*}\right)^{2} \boldsymbol{\Sigma}_{2}-\sigma^{2} E_{2}^{*} \boldsymbol{\Sigma}_{1}+\sigma^{2} E_{1}^{*} \boldsymbol{\Sigma}_{2}\right) \mathbf{w}_{T}^{*},
$$

and

$$
\mathbf{b}_{2}=\left(\mathbf{I}-\mathbf{w}_{T}^{*}\left(\mathbf{w}_{T}^{*}\right)^{H}\right)\left(\left(E_{2}^{*}\right)^{2} \boldsymbol{\Sigma}_{1}\right) \mathbf{w}_{T}^{*} .
$$

\section{EXAMPLES}

In this section we present some examples that illustrate the achievable rate region for a two-user $4 \times 4$ RF-MIMO BC. The entries of $\mathbf{H}_{i}(i=1,2)$ are i.i.d. zero-mean complex Gaussian random variables with variance $\sigma_{h_{i}}^{2}$, which is chosen to obtain a given $S N R_{i}=10 \log _{10} \sigma_{h_{i}}^{2} / \sigma^{2}$ per receive antenna. The channel coefficients are kept fixed (no fading) and, without loss of generality, we take $\sigma^{2}=1$.

To get the boundary of achievable rate pairs we follow the procedure described in Section IV, which is summarized here as follows:

1) For a dense grid obtained by varying $\alpha$ between 0 and 1, the optimal transmit beamformers, $\mathbf{w}_{T}^{*}$, are computed as the eigenvector corresponding to the largest eigenvalue of $\boldsymbol{\Sigma}_{\alpha}=$ $\alpha \boldsymbol{\Sigma}_{1}+(1-\alpha) \boldsymbol{\Sigma}_{2}$.

2) For each $\mathbf{w}_{T}^{*}$, obtain the energies of the equivalent SISO channels after optimal Tx-Rx beamforming: $E_{1}^{*}=$ $\left(\mathbf{w}_{T}^{*}\right)^{H} \boldsymbol{\Sigma}_{1} \mathbf{w}_{T}^{*}$ and $E_{2}^{*}=\left(\mathbf{w}_{T}^{*}\right)^{H} \boldsymbol{\Sigma}_{2} \mathbf{w}_{T}^{*}$; and then check which user has a better channel. If $E_{1}^{*}>E_{2}^{*}$ the optimal power allocated to user 1 is given by (11); otherwise it is given by (15).

The proposed procedure to obtain the optimal transmit beamformers yields SISO channels that lie on the Pareto boundary of the energy region $\left(E_{1}, E_{2}\right)$. However, it might happen that, for some of the pairs $\left(\mathbf{w}_{T}^{*}, P^{*}\right)$, the corresponding rates fall below the boundary of the achievable region. This can be easily explained because, as Theorem 1 proves, all points on the $\left(R_{1}, R_{2}\right)$ boundary map into the $\left(E_{1}, E_{2}\right)$ boundary, but the converse is not necessarily true. Nevertheless, since the rate boundary is completely characterized by the proposed procedure, those points (if any) that fall below the boundary can easily be discarded in a final step.

\section{V-A. Asymmetric downlink channel}

For the first example we consider an asymmetric downlink channel in which $S N R_{1}=10 \mathrm{~dB}$ and $S N R_{2}=0 \mathrm{~dB}$. Fig. 5 shows the Pareto boundary obtained with the proposed procedure in black solid line. The achievable rate regions using DET for both users are also shown in dashed lines. For this example, when $\alpha$ varies from 0 to 1 , the equivalent SISO channel of user 1 is always better than that of user $2\left(h_{1}>h_{2}, \forall \alpha\right)$. We refer to this kind of channels as RF-MIMO degraded broadcast channels. In this 


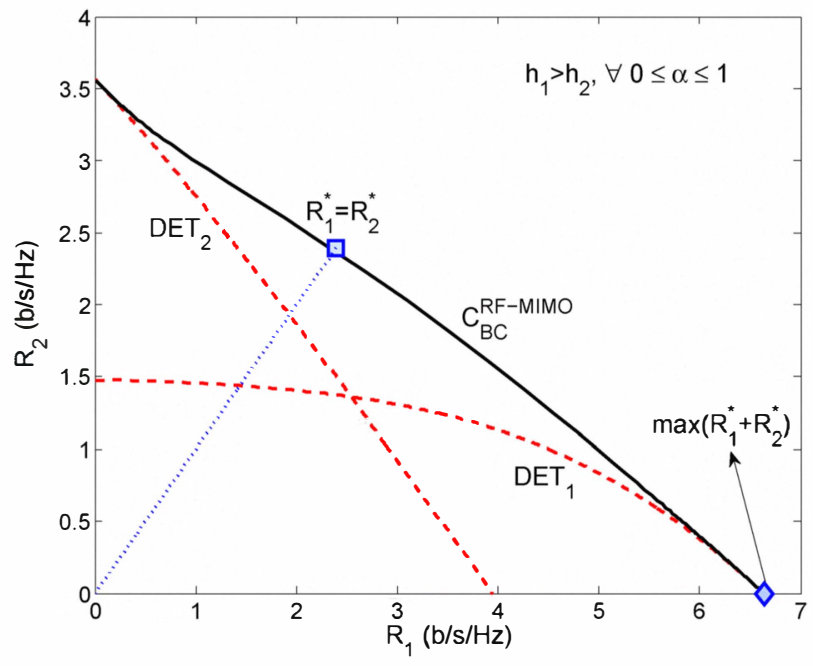

Fig. 5. $C_{B C}^{R F-M I M O}$ boundary in black solid line for an asymmetric two-user $4 \times 4$ downlink channel $\left(S N R_{1}=10 \mathrm{~dB}\right.$ and $S N R_{2}=0$ $\mathrm{dB})$. In this case, any value $0 \leq \alpha \leq 1$ yields a point in the boundary. The red dashed lines indicate the DET boundary for both users. The egalitarian and the max sum-rate solutions are marked with a square and a diamond, respectively.

situation, we experimentally observe that for any $0 \leq \alpha \leq 1$ the corresponding $\left(\mathbf{w}_{T}^{*}, P^{*}\right)$ always yields a pair of rates on the boundary. This seems to be always the case when one of the users has a stronger channel than the other for any capacity achieving Tx beamformer. More formally, we conjecture that for an RF-MIMO degraded $\mathrm{BC}$ the mapping between the Pareto optimal boundaries of energies and rates is bijective (a proof is yet to be found).

Based on the characterization of the Pareto boundary, we can easily find the solutions $\left(\mathbf{w}_{T}^{*}, P^{*}\right)$ that provide interesting pair rates. A typical example is the maximum sum-rate solution, which in this case is obtained for $\alpha=1$ and consists of transmitting all available power to user $1\left(P^{*}=1\right)$ through the maximum eigenvector of $\boldsymbol{\Sigma}_{1}=\mathbf{H}_{1}^{H} \mathbf{H}_{1}$ (i.e., dominant eigenmode transmission for the best user). This point is marked with a diamond in Fig. 5. The rate of user 2 is obvioulsy $R_{2}=0$.

Another interesting operating point is the max-min optimal pair, which is sometimes called the egalitarian solution and is given by the crossing point between the Pareto boundary and the line $R_{1}=R_{2}$. This point, marked with a square in Fig. 5, is achieved by a Tx beamformer that maximizes (4) for $\alpha=0.0965$, and using superposition coding with $P^{*}=0.0764$.

\section{V-B. Symmetric downlink channel}

In the second example we consider a symmetric downlink scenario in which both users have the same $S N R=10 \mathrm{~dB}$. Repeating the procedure, we now find that the transmit beamformers obtained by maximizing (4) are only capacity achieving for $\alpha \in \Theta=\{[0,0.136) \cup(0.443,1]\}$. The results are shown in Fig. 6, where the rates corresponding to solutions obtained for $\alpha \in[0.136,0.443]$ are shown in dashed line. Remember that these values yield beamformers that are Pareto optimal of the region $\left(E_{1}, E_{2}\right)$, but are not optimal in terms of rates. Since the boundary of rate pairs is perfectly characterized by the rest of $\alpha$ 's, these points can be easily discarded by visual inspection. For this example the channel is not degraded after RF-MIMO beamforming, which means that for some points of the boundary $h_{1}>h_{2}$, while for others $h_{2}>h_{1}$. Therefore, the ordering between users is not maintained when we move along the boundary.

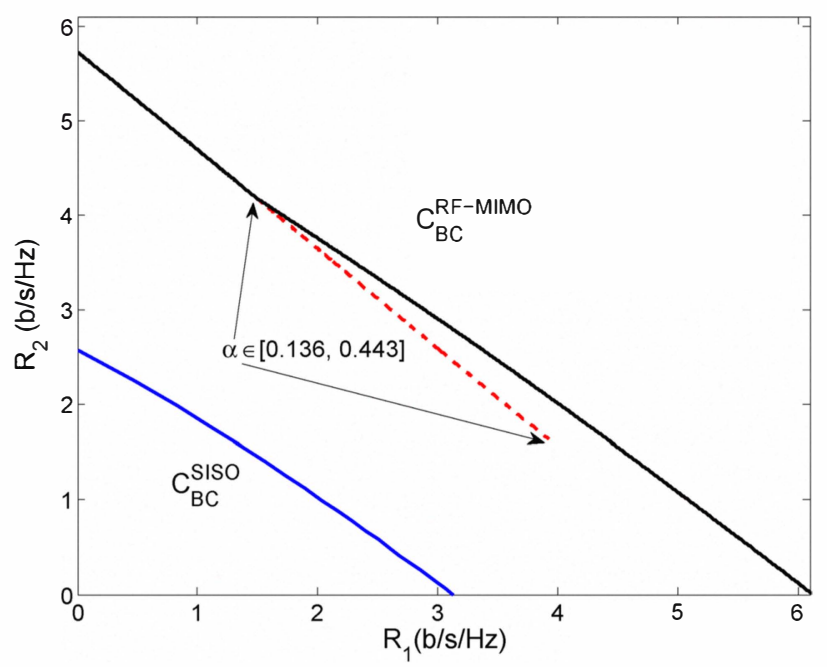

Fig. 6. $C_{B C}^{R F-M I M O}$ boundary for a symmetric two-user $4 \times 4$ downlink channel $\left(S N R_{1}=10 \mathrm{~dB}\right.$ and $\left.S N R_{2}=10 \mathrm{~dB}\right)$. In this case, the rate pairs corresponding to $0.136 \leq \alpha \leq 0.443$ (in dashed line) are not on the boundary. For comparison the rate region for a SISO BC using the first antenna of each terminal is also shown.

Fig. 6 also shows the rate region for the SISO channel obtained by selecting the first antenna at the transmitter and at both receivers. We clearly observe the enlargement of the capacity region due to Tx-Rx analog beamforming.

\section{EXTENSIONS}

\section{VI-A. MAC-BC duality}

Like in many other channel models [18], there also exists a duality relationship between the $\mathrm{BC}$ and the multiple access channel (MAC) when all terminals have multiple antennas and use analog beamforming. More specifically, suppose that a point in the BC boundary is achieved by using superposition coding with a transmit beamformer $\mathbf{w}_{T}^{*}$, two receive maximum ratio beamformers $\left(\mathbf{w}_{R_{1}}^{*}, \mathbf{w}_{R_{2}}^{*}\right)$ given by (1), and a power $P^{*}$ allocated to user 1 . If we consider now a dual MAC (uplink) with reciprocal MIMO channels $\mathbf{H}_{1}^{H}$ and $\mathbf{H}_{2}^{H}$, with the same total power constraint and the same noise statistics; then the same beamformers are capacity achieving if we reverse the roles of transmitter and receivers. That is, for the dual MAC the optimal transmit beamformers are $\mathbf{w}_{R_{1}}^{*}$ and $\mathbf{w}_{R_{2}}^{*}$, and the base station must apply successive cancellation after performing receive beamforming with $\mathbf{w}_{T}^{*}$. It should be emphasized, however, that the powers in the MAC channel to achieve a given point in the BC boundary are not the same. As an example, Fig. 7 shows the boundary for a 2-user RF-MIMO $\mathrm{BC}$ where the users have a $10-\mathrm{dB}$ difference in gain. The square marker indicates a particular point obtained for $\alpha=0.15$ and $P^{*}=0.1960$. For the dual MAC, if we use the same beamformers and vary the power between users subject to the same total power constraint $P_{1}+P_{2}=1$, we obtain the achievable rate regions shown in dashed line ${ }^{6}$. In particular, using $P_{1}=0.7001$ in the dual MAC, we obtain the same rates as in the BC channel with $P^{*}=0.1960$.

\section{VI-B. The K-user case}

The results for the 2-user case can easily be extended to a $\mathrm{K}$ user system. For simplicity, let us assume an RF-MIMO BC with

${ }^{6}$ For the MAC, the straight line connecting the two corner points can be obtained not only with time sharing (which is not possible with our RFMIMO architecture), but also with a technique called rate splitting [19] 


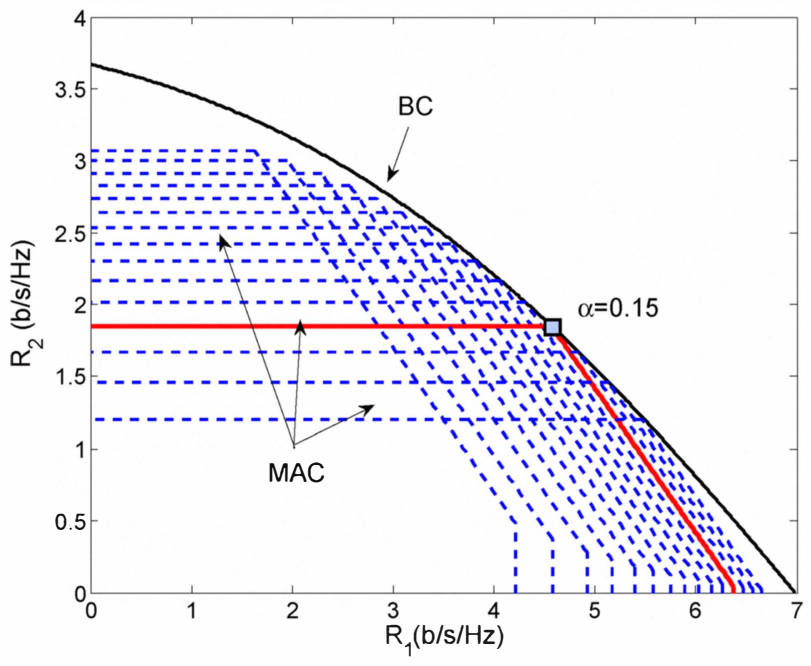

Fig. 7. Duality between the $\mathrm{BC}$ and MAC with Tx-Rx analog beamforming. The Tx-Rx beamformers obtained for $\alpha=0.15$ (marked with a square) are capacity achieving for the BC and the dual MAC. To achieve the point on the boundary, user 1 in the BC transmits with power $P^{*}=0.1960$, whereas in the MAC the power of user 1 is $P_{1}=0.7001$.

3 users. The optimal transmit beamformer can be obtained in this case by maximizing the weighted sum-energy cost function

$$
\max _{\mathbf{w}_{T}} \alpha_{1} E_{1}+\alpha_{2} E_{2}+\alpha_{3} E_{3}
$$

where the weights belong to a three-dimensional simplex: $\alpha=$ $\left\{\left(\alpha_{1}, \alpha_{1}, \alpha_{3}\right) \in \mathbb{R}^{3} \mid \alpha_{i} \geq 0, \sum_{i=1}^{3} \alpha_{i}=1\right\}$. The problem remains to get the optimal power allocation $\left(P_{1}^{*}, P_{2}^{*}, P_{3}^{*}\right)$ subject to a unitpower constraint. Let us simply sketch the procedure: assume that $\mathbf{w}_{T}^{*}$ is a maximizer of (16) that results in $E_{1}^{*}>E_{2}^{*}>E_{3}^{*}$. Using superposition coding and successive cancellation the following triplet of rates can be achieved: $R_{1}=\log _{2}\left(1+P_{1} E_{1}^{*} / \sigma^{2}\right)$, $R_{2}=\log _{2}\left(1+P_{2} E_{2}^{*} /\left(P_{1} E_{2}^{*}+\sigma^{2}\right)\right)$ and $R_{3}=\log _{2}(1+$ $\left.P_{3} E_{3}^{*} /\left(P_{1} E_{3}^{*}+P_{2} E_{3}^{*}+\sigma^{2}\right)\right)$. Again, Pareto optimality can be applied to obtain the optimal power allocation. Specifically, we fix the rates of two users (e.g., $R_{1}$ and $R_{2}$ ) and equate to zero the derivative of $R_{3}$ with respect to $\mathrm{w}_{T}^{*}$. Repeating the procedure for other pairs of rates, we obtain a system of equations from which the optimal power distribution can be obtained. For brevity, details are omitted here.

\section{CONCLUSIONS}

RF-MIMO wireless radios performing analog beamforming in the RF domain result in transceivers with reduced system size, costs, and power consumption. In this paper we have studied the achievable rate region of the RF-MIMO two-user broadcast channel. In particular, any Pareto point on the boundary can be achieved by transmitting through a given direction and using superposition coding. Our main contribution has been to show that the optimal transmit beamformer and the optimal power allocation can be obtained in closed form using a computationally simple procedure. These results can be generalized to the MAC (using duality arguments), as well as to the $\mathrm{K}$-user $\mathrm{BC}$. Considering fading or frequency-selective channels are interesting extensions to continue this work.

\footnotetext{
${ }^{7}$ Theorem 1 can also be generalized to a K-user RF-MIMO broadcast channel.
}

\section{REFERENCES}

[1] R. Eickhoff, R. Kraemer, I. Santamaria, L. Gonzalez, "Developing energy-efficient MIMO radios," IEEE Vehicular Technology Magazine, vol. 4, no. 1, pp.34-41, Marzo 2009.

[2] I. Santamaria et al, "Optimal MIMO transmission schemes with adaptive antenna combining in the RF path," 16th European Signal Processing Conference, (EUSIPCO 2008), Lausanne, Switzerland, Aug. 2008.

[3] J. Via, I. Santamaria, V. Elvira, R. Eickhoff, "A general criterion for analog Tx-Rx beamforming under OFDM transmissions," IEEE Trans. Signal Proc., vol.58, no.4, pp. 21552167, April 2010.

[4] J. Via, V. Elvira, I. Santamaria, R. Eickhoff, "Analog antenna combining for maximum capacity under OFDM transmissions," IEEE Int. Conf. on Comm., (ICC 2009), Dresden, Germany, June 2009.

[5] J. Via, V. Elvira, I. Santamaria, R. Eickhoff, “ Minimum BER beamforming in the RF domain for OFDM transmissions and linear receivers," IEEE Int. Conf. on Acoust. Speech and Sig. Proc. (ICASSP 2009), Taipei, Taiwan, Abril 2009

[6] T. M. Cover, J. A. Thomas, Elements of Information Theory, John Wiley, New York, 1991.

[7] P. P. Bergmans, T. M. Cover, "Cooperative broadcasting," IEEE Trans. Inf. Theory, pp. 317-324, May 1974.

[8] H. Weingarten, Y. Steinberg, and S. Shamai, " The capacity region of the Gaussian multiple-input multiple-output broadcast channel," IEEE Trans. Inf. Theory, vol. 52, no. 9, pp. 39363964, Sept. 2006.

[9] G. Caire, S. Shamai, "On the achievable throughput of a multiantenna Gaussian broadcast channel," IEEE Trans. Inf. Theory, pp. 1691-1706, July 2003.

[10] F. Ellinger, Radio Frequency Integrated Circuits and Technologies, New York: Springer-Verlag, 2007.

[11] F. Rashid-Farrokhi, L. Tassiulas, K. J. Ray Liu, "Joint optimal power control and beamforming in wireless networks using antenna arrays," IEEE Trans. Commun., vol. 46, no. 10, pp. 1313-1706, Oct. 1998.

[12] M. Schubert, H. Boche, "Solution of the multiuser downlink beamforming problem with individual SINR constraints," IEEE Trans. Veh. Technol., vol. 53, no.1, pp. 18-28, Jan 2004.

[13] D. Tse, P. Viswanath, Fundamentals of Wireless Communication, Cambridge University Press, 2005.

[14] A. Goldsmith, Wireless Communications, Cambridge University Press, 2005.

[15] J. B. Andersen, "Array gain and capacity for known random channels with multiple element arrays at both ends," IEEE $J$. on Select. Areas Commun., vol. 11, pp. 21722178, Nov. 2000.

[16] S. Boyd, L. Vandenberghe, Convex Optimization, Cambridge University Press, 2004.

[17] A. Edelman, T. Arias, S. T. Smith, "The geometry of algorithms with orthogonality constraints," SIAM J. Matrix Anal. Appl., vol. 20, pp. 303-353, 2008.

[18] N. Jindal, S. Vishwanath, A. Goldsmith, "On the duality of Gaussian multiple-access and broadcast channels," IEEE Trans. Inf. Theory, pp. 768-783, May 2004.

[19] B. Rimoldi, R. Urbanke, "A rate-splitting approach to the Gaussian multiple-access channel," IEEE Trans. Inf. Theory, pp. 364-375, March 1996. 